Original Article

\title{
Determination of efficacy of pre-procedural mouth rinsing in reducing aerosol contamination produced by ultrasonic scalers
}

\author{
Reema M. Rao ${ }^{1}$, Nina Shenoy ${ }^{2} \&$ Veena Shetty ${ }^{3}$ \\ ${ }^{1}$ Postgraduate, ${ }^{2}$ Professor, Department of Periodontics, A. B. Shetty M emorial Institute Of Dental Sciences, ${ }^{3}$ Professor, \\ Department of M icrobiology, K.S. Hegde M edical Academy, Nitte University, M angalore, Karnataka, India. \\ Correspondence \\ Reema M. Rao \\ Post Graduate, Department of Periodontics , A.B. Shetty Memorial Institute of Dental Sciences, \\ Nitte University , M angalore - 575 018, Karnataka, India. \\ Mobile : +91 9481844347 E-mail : reemamrao1@gmail.com
}

\begin{abstract}
The oral cavity is a unique environment which can provide an ideal medium for bacterial growth. M ost of the procedures performed by dentist have the potential for creating contaminated aerosols and splatter which contains bacteria, fungi, protozoa and even blood borne viruses produced during dental operative procedures and thus promoting an increased risk of cross infection. In dentistry, the ultrasonic scaler and the air polisher are considered to be the greatest producers of small particle aerosol contamination. Aerosols can remain airborne for extended periods of time and may be inhaled and was also found that the microorganisms could survive in the aerosol produced for as long as 6 days. The association of these aerosols with the respiratory infections, opthalamic and skin infections, tuberculosis and hepatitis $B$ have been reported.

Chlorhexidine is considered as the "Gold standard" of antimicrobial rinse because of broad-spectrum antibacterial activity and substantivity of 8-12 hrs. This study determined the efficacy of preprocedural rinsing with an antimicrobial mouthrinse containing chlorhexidine in reducing the level of viable bacteria contained in aerosols generated by ultrasonic scaling.

The results of the present study clearly indicate that pre-procedural mouth rinse with $0.2 \%$ chlorhexidine gluconate was significantly effective in reducing the aerosol contamination during use of ultrasonic scaling in dental practice Using a pre-procedural rinse can significantly reduce the viable microbial content of dental aerosols.
\end{abstract}

Keywords: Aerosols, ultrasonic scaling, colony formingunits

\section{Introduction}

The oral cavity is a unique environment which can provide an ideal medium for bacterial growth. Most of the procedures performed by dentist have the potential for creating contaminated aerosols and splatter which contains bacteria, fungi, protozoa and even blood borne viruses produced during dental operative procedures and thus promoting an increased risk of cross infection. ${ }^{1}$ The use of ultrasonic scalers, prophy angles, and air-water syringes produce some Access this article online Quick Response Code

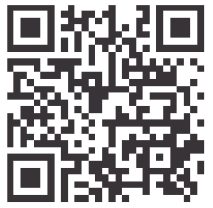
splatter in the form of relatively large droplets. ${ }^{2}$ In dentistry, the ultrasonic scaler and the air polisher are considered to be the greatest producers of small particle aerosol contamination. Ultrasonic scaler considered to produce more airborne contamination than any other instruments in dentistry. In dental clinic, dentists and patients are daily exposed to a great variety of infectious agents and toxic substancestransported by aerosols and droplets.

Micik et al proposed the terminology, "aerosol and splatter" in dental environment and are considered to be the pioneers in the work on aerobiology, where patient is the source and the aerosols originates from the saliva as well as from the dental plaque. ${ }^{2}$ Aerosols are suspensions of liquid and or solid particles in the air consists of particles less than 10 microns in diameter which are not visible to the naked eye. ${ }^{3}$ When the ultrasonic tip comes into contact with fluids it leads to aerosol formation. The fluid may be saliva, blood or water, the energy transmitted to the fluid is enough to produce the aerosol suspension of fine liquid particles. The ultrasonic scaler tip produces both small and 
large particles. The use of rotary dental and surgical instruments like handpieces, ultrasonic scalers and airwater syringes can produce visible spray which are called as splatter and can travel only a short distance and settles out quickly either on the floor, nearby equipment and operatory surfaces, the dentist and or on the patient. ${ }^{4}$ The spray may also contain some aerosol. Aerosols can remain airborne for extended period of time and may be inhaled and was also found that the microorganisms could survive in the aerosol produced for as long as 6 days. ${ }^{5}$

The smaller particles of an aerosol (0.5 to $10 \mu \mathrm{m}$ in diameter) have the potential to penetrate and lodge in the smaller passages of the lungs and carry the greatest potential for transmitting infections. ${ }^{2}$ According to the study conducted by Millers, aerosols generated from the patients mouth contains upto $1,00,000$ bacteria per cubic foot of air. ${ }^{6}$ The association of these aerosols with the respiratory infections, opthalamic and skin infections, tuberculosis and hepatitis B have been reported in other studies. ${ }^{6}$ Several methods like using high vacuum suction, patient positioning, use of rubber dams, and pre-procedural antibacterial mouth rinses have been tried to reduce aerosol contamination in the dental office. ${ }^{5}$ Current literature suggests that having patients use an antimicrobial rinse before treatment may decrease microbial aerosols.

Chlorhexidine is considered as the "Gold standard" of antimicrobial rinse because of broad-spectrum antibacterial activity and substantivity of $8-12 \mathrm{hrs} .{ }^{3,5}$

With this background the aim of this study was to determine the efficacy of preprocedural rinsing with an antimicrobial mouthrinse containing chlorhexidine in reducing the level of viable bacteria contained in aerosols generated by ultrasonic scaling.

\section{Materialsand method}

30 subjects were selected from the Department of Periodontics, A.B. Shetty Memorial Institute of Dental Sciences, Deralakatte, Mangalore. After obtaining the ethical clearance, written informed consent was taken from all the participants before start of the study and were divided into two groups i.e. group I and group II .Group I included 15 subjects with moderate form of chronic periodontitis undergoing ultrasonic scaling without pre procedural rinsing and Group II included 15 subjects with moderate form of chronic periodontitis undergoing ultrasonic scaling with pre procedural rinsing with an antimicrobial mouthwash $(10 \mathrm{ml}$ of undiluted $0.2 \%$ of Chlorhexidine mouth wash).

Gingival index according to criteria given by Loe and Silness (1963) was recorded.

Full mouth periodontal examination was recorded which included the determination of clinical attachment level using William graduated periodontal probe.

\section{INCLUSION CRITERIA}

- Subjects aged between 25 to 55 years.

- Subjects with a minimum complement of 20 teeth.

- Subjects who were diagnosed with moderate form of chronic periodontitis showing more than $30 \%$ of sites with clinical attachment loss $\geq 4 \mathrm{~mm}$ measured with a Williams periodontal probe.?

\section{EXCLUSION CRITERIA:}

- Subjects with any known systemic disease or conditions.

- Patients who underwent any periodontal treatment in the last 6 months.

- Patients who are already using mouth washes.

- History of any antibiotic /anti-inflammatory therapy for three months prior to study.

- Pregnant and lactating women.

- Patientswith dentinal hypersensitivity.

- Patients hypersensitive to chlorhexidine mouth wash.

\section{METHOD OF COLECTION OF SAM PLE}

- Blood agar plate was used to collect the airborne microorganisms as it is valid medium for culturing airborne bacteria. Out of the two blood agar plates, one plate was positioned at the patients chest area and other at the dentist chest area with the help of double 
sided adhesive tape. The average distance was approximately 12 inches from the patient's mouth to agar plate. Scaling was carried out with piezoelectric ultrasonic scaler during each treatment and a motorized suction was used for every patient. Each treatment session consisted of ultrasonic scaling which was of 30 minutes duration. 15 of 30 patients rinsed with $10 \mathrm{ml}$ of undiluted $0.2 \%$ Chlorhexidine mouth wash ten minutes before the treatment.

- Blood agar plate was left uncovered at predesigned sites to collect the samples of aerosolized bacteria. After collecting the sample, the blood agar plates were incubated at 37 degree Celsius for 48hrs. The evaluation of the number of Colony forming units that grew on the each plate was done in Microbiology department,K S Hegde Medical Academy.

\section{Statistical analysis}

All the samples collected were subjected to statistical analysis

Comparison of the two groups was done using Mann Whitney U test

\section{Results}

The comparison of the two groups indicates that there is higher median values in the non mouthwash category. The number of colonies forming units formed on blood agar plates were less in patients with preprocedural rinse with mouth wash than the patients who had not used the mouth wash as shown in table. The highest number of colonies was found on blood agar plate positioned at the patient's chest area followed by the doctors. The results showed that CFU in group II were significantly reduced when compared to group I with the $p$ vaule $\varangle 0.001$, which was statistically significant (table).

\section{Discussion}

Dental plaque is considered as one of the etiological agent in the development of periodontal disease comprising complexes of microorganisms, both bacterial and viral origin in the gelatinous matrix. ${ }^{1}$ Dental calculus is porous in nature and it can absorb various toxic products that can damage the periodontal tissues. Hence, calculus should be accurately detected and thoroughly removed for adequate periodontal therapy. Conventional non-surgical therapy is considered to be the cornerstone of periodontal treatment. ${ }^{7,8}$ It includes hand instruments and ultrasonic scaling. Ultrasonics produce high frequency vibrations which leads to phenomenon of cavitation, micro streaming. This phenomenon aids in disruption of bacterial cell wall as well as calculus removal. Along with advantages of ultrasonics, there are some disadvantages like it is not tactile sensitive and it leads to production of large amount of aerosols.

The ultrasonic scalers produces aerosols that are heavily contaminated by the microorganisms and can cause a serious health threat to the patients, clinician and the surrounding, in the form of systemic conditions like common cold, influenza, tuberculosis and severe acute respiratory syndrome (SARS). ${ }^{1}$ As the oral pathogens show a high probability of bypassing the host defense, to reduce the bacterial load in the aerosol, adjunct therapy in the form of chemical plaque control is required. Studies have also shown that that ultrasonic scaling in conjunction with various plaque control agents used as a pre-procedural rinse have been found to be more effective in reducing bacterial loads when compared with distilled water or saline. .,9 $^{5}$

Various mouth washes like Listerine, Peridex, Clorhex plus has been used in reducing the aerosol count while performing ultrasonic scaling. But, chlorhexidine is recognized as the Gold standard for chemical plaque control because of good substantivity., ${ }^{3,5,10,11}$

Chlorhexidine is a bisbiguanide molecule that binds strongly to hydroxyapatite, the organic pellicle of the tooth, oral mucosa, salivary proteins, and bacteria. Because of this binding, chlorhexidine containing mouthrinses exhibit high substantivity with $30 \%$ of drug released after rinsing and slow release for long time. $0.2 \%$ of Chlorhexidine was the first clinically effective and demonstrated mouthwash, that inhibited supragingival plaque formation and due to 
broad spectrum antimicrobial activity of chlorhexidine, it is highly effective against gram positive organisms, gram negative organisms, yeasts, dermatophytes and some lipophilic viruses. ${ }^{5,10,13}$

The results in several studies have indicated that chlorhexidine mouth wash is superior to essential oil mouth washes. .,10 $^{-10}$

In the present study, blood agar plates were used to collect the airborne microorganisms as it considered to be a valid non selective medium for culturing airborne bacteria. When an aerobic bacterium settles and grows as a colony, it is counted as colony forming units (cfu).The total number of colony forming units formed was counted on blood agar plates.

This study revealed that both the dentist and the patient were exposed to high amounts of bacteria due to aerosols produced by ultrasonic scaling. The highest number of colonies was seen on the plates positioned on patient's chest area (Graph 1). The larger salivary droplets generated during dental procedures settle down rapidly from the air with heavy contamination of a plates placed at patient's chest area. This was followed by the contamination on blood agar placed at operator's chest area which was placed 12 inches from the operating area (Graph II). When $10 \mathrm{ml}$ of undiluted $0.2 \%$ of chlorhexidine was used as a pre procedural mouth rinse prior to ultrasonic scaling, fewer colony-forming units developed than the ultrasonic scaling done without the use of mouth wash. These results were in accordance with other studies in which blood agar plate positioned at patients chest area received a greater number of microorganisms and demonstrated the efficacy of pre-procedural rinsing with chlorhexidine in reducing the aerosol contamination produced by ultrasonic scaling. Use of $0.2 \%$ chlorhexidine gluconate mouthwashes as a pre procedural mouth rinsing for the duration of 60 seconds can cause substantial reduction in bacterial counts. ${ }^{1,3,5}$

However a study was reported in the literature which revealed that herbal mouth rinse produced the largest zones of microbial inhibition when compared to Listerine and $0.12 \%$ Chlorhexidine. $^{14}$

The use of high volume evacuator attachment and aerosol reduction device can synergistically aid in effective reduction of aerosol contamination without increased heat transfer to the tooth and is effective in reducing the number of microorganisms generated during ultrasonic scaling, therefore decreasing the risk of disease transmission. ${ }^{15,16}$

The results of the present study clearly indicate that preprocedural mouth rinse with $0.2 \%$ chlorhexidine gluconate was significantly effective in reducing the aerosol contamination during use of ultrasonic scaling in dental practice. Various other studies support the results of this study demonstrating the excellent antimicrobial effects of $0.2 \%$ chlorhexidine as a pre procedural mouth rinse in aerosol reduction. ${ }^{17,18}$

\section{COM PARISON OF THE TWO GROUPSUSING MANN WHITNEY UTEST: IN PATIENT AND DENTISTS}

$M$ ann Whitney $U$ test is a non-parametric test which is used to compare the efficacy of pre procedural mouth rinse before ultrasonic scaling.

\begin{tabular}{|l|l|c|c|c|c|c|c|c|c|c|c|}
\hline \multicolumn{2}{|c|}{} & N & $\begin{array}{c}\text { M inimum } \\
\text { CFU }\end{array}$ & $\begin{array}{c}\text { Maximum } \\
\text { CFU }\end{array}$ & 25 & M EDIAN & 75 & $\begin{array}{c}\text { Sum of } \\
\text { Ranks }\end{array}$ & $\begin{array}{c}\text { M ann- } \\
\text { Whitney U }\end{array}$ & $\begin{array}{c}\text { Z } \\
\text { Exact Sig. } \\
(2 \text {-tailed) }\end{array}$ \\
\hline Patients & $\begin{array}{l}\text { Patient without } \\
\text { mouthwash }\end{array}$ & 15 & 6 & 11 & 8 & $\underline{\mathbf{8}}$ & 9 & 342.5 & 2.5 & - & $\underline{\mathbf{0 0 . 0 0 1}}$ \\
\hline $\begin{array}{l}\text { Patient with } \\
\text { mouthwash }\end{array}$ & 15 & 1 & 7 & 3 & $\underline{\mathbf{3}}$ & 4 & 122.5 & & & \\
\hline Dentist & $\begin{array}{l}\text { Patient without } \\
\text { mouthwash }\end{array}$ & 15 & 4 & 7 & 4 & $\underline{\mathbf{5}}$ & 6 & 343 & 2 & -4.635 & $\underline{\mathbf{0 0 . 0 0 1}}$ \\
\hline & $\begin{array}{l}\text { Patient with } \\
\text { mouthwash }\end{array}$ & 15 & 0 & 4 & 1 & $\underline{\mathbf{2}}$ & 2 & 122 & & & \\
\hline
\end{tabular}

The comparison of the two groups indicates that there is higher median values in the non-mouthwash category 


\section{Graph}

This error plot graph indicates the comparison of patients with and without mouthwash. The upper tail and lower tail of graphs indicates the maximum and minimum number of colony forming units on the blood agar plates placed on the patients and dentists chest area. The central dark line inside the box is the median, the upper and lower end of the box is $75^{\text {th }}$ and the $25^{\text {th }}$ percentile, the tails represent the maximum and the minimum values.

Graph 1: Comparison of colony forming units on the blood agar plate positioned at patients chest area without and with mouth wash

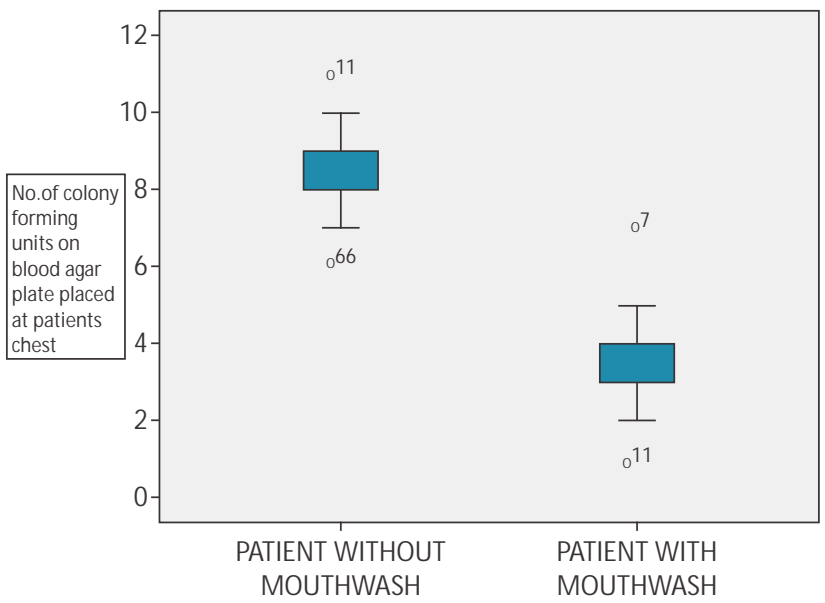

\section{References}

1. SwaninathanY.Thomas JT, Muralidharan NP. The Efficacy Of Preprocedural M outh Rinse Of $0.2 \%$ Chlorhexidine And Commercially Available Herbal M outh Containing SalvadoraPersica In Reducing The Bacterial Load In Saliva And Aerosol Produced During Scaling Asian J Pharm Clin Res, 2014; Vol 7, Suppl 1: 71-74.

2. Acharya S,Priya H, Purohit B, Bhat M. Aerosol contamination in a Rural University Dental Clinic in South India. Int J Infect Control 2010, Vol 6, $1-7$

3. Suresh S, M animegalai S, Sudhakar u, Sophia Comparison of Efficacy of Preprocedural Rinsing with Chlorhexidine and Essential Oil Mouthwash in Reducing Viable Bacteria In Dental Aerosols-A Microbiological Study . Int. Journal of Contemporary Dentistry 2011; 2(6):1-6

4. Bentley CD, Burkhart NW, Crawford JJ. Evaluating spatter and aerosol contamination during dental procedures. J Am Dent Assoc1994; 125(5): 579-584.

5. Reddy S, Prasad MGS, Kaul S, Satish K Efficacy of $0.2 \%$ tempered chlorhexidine as a preprocedural mouth rinse: A clinical study .J Indian SocPeriodontol. 2012 Apr-Jun; 16(2): 213-217.

6. Gupta G, Mitra D, Ashok, Soni S Comparison of Efficacy of PreProcedural Mouth Rinsing in Reducing Aerosol Contamination Produced by Ultrasonic Scaler: A Pilot Study . J Periodontol 2014:85:562-568.

7. Caranza's Clinical Periodontology :Armitage GC ,Classification of periodontal diseases and conditions, tenth edition,7, page101.

8. Deepa G. Kamath, Nayak S. Detection, removal and prevention of calculus:Literature Review The Saudi Dental Journal 2014 ; 26(1): 7-13.

9 Fine DH, M endieta C, Barnett M L, Furgang D, M eyers R, Olshan A and Vincent J..Efficacy of preprocedural rinse with an antiseptic in reducing
Graph II: Comparison of colony forming units on the blood agar plate positioned at dentist chest area with and without mouth wash

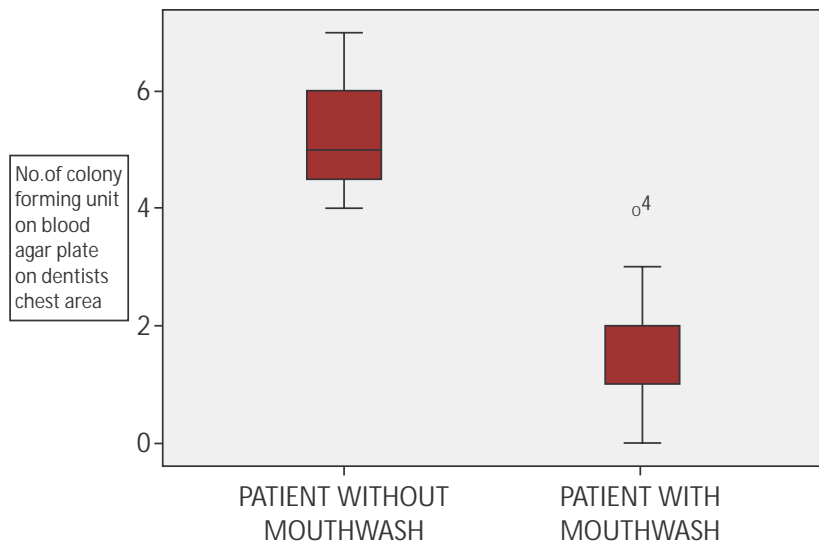

\section{Conclusion}

In the present study conducted, we found that $0.2 \%$ of chlorhexidine had a significant effect as an antimicrobial pre-procedural mouth rinse in reducing the number of microorganisms in the aerosol produced by the ultrasonic scaling units. Using a pre-procedural rinse can significantly reduce the viable microbial content of dental aerosols. Use of blood agar plates prior to and after ultrasonic scaling and evaluating the comparison between other commercially available mouth washes were limitations of the study.

viable bacteria in dental aerosol.J Periodontal 1992;63:821-24.

10. Logothetis DD, Martinez-Welles JM. Reducing bacterial aerosol contamination with a chlorhexidine gluconate pre-rinse. J Am Dent Assoc 1995; 126: 1634-1639.

11. Son W-K, Shin S-Y, Kye S-B, Yang S-M. The effect of chlorhexidine on reduction of viable organisms in aerosol produced by ultrasonic scaler. J Korean AcadPeriodontol 2009;39:303-310.

12. Walsh TF, Unsal E, Davis LG, Yilmaz O. the effect of irrigation with chorhexidine or saline on plaque vitality. J Clin Periodontal 1995;22:262-4.

13.S antos A. Evidence-based control of plaque and gingivitis. ClinPeriodontol 2003;30Suppl 5:13-6.

14. A M Khalessi, A R C Pack, W M Thomson, G R Tompkins: An in vivo study of the plaque control efficacy of Persica : a commercially available herbal mouthwash containing extracts of Salvadorapersica. International Dental Journal 2004; 54(5): 279-283.

15. Harrel SK, Barnes) and Hidalgo FR.J Reduction of Aerosols Produced by Ultrasonic Scalers.J Periodontol 1996;67:28-32.

16. King BT, M uzzin K, Berry CW and Anders L . The Effectiveness of an Aerosol Reduction Device for Ultrasonic Scalers. J Periodontol 1997;68:45-49.

17. Woo -Kyung Son, Seung-Yun Shin, BeomKye and Seung-M in Yang.The effect of chlorhexidine on reduction of viable organisms in aerosol produced by ultrasonic scaler.J Korean AcadPeriodontol 2009; 39(3):303-310.

18. Bharathi Purhohit, Harsh Priya. Efficacy of preprocedural rinsing in reducing aerosol contamination during dental procedures.Journal of Infection Prevention 2009; vol 10 (6): 190-192. 